\title{
Perceptions of Traumatic Brain Injury (TBI) Among Professionals Providing Drug and Alcohol Addiction Treatment
}

\author{
Jerrod Brown ${ }^{1,2,3 *}$, Diane Harr' \\ 'Concordia University, St. Paul, MN, USA \\ ${ }^{2}$ Pathways Counseling Center, Inc., St. Paul, MN, USA \\ ${ }^{3}$ American Institute for the Advancement of Forensic Studies, St. Paul, MN, USA
}

Article Info

Article Notes

Received: April 3, 2019

Accepted: May 6, 2019

\section{${ }^{*}$ Correspondence:}

Dr. Jerrod Brown, Ph.D. Pathways Counseling Center, Inc., 1919 University Avenue West Suite 6 St. Paul, Minnesota, 55104, USA; Email: jerrod01234brown@live.com.

(c) 2019 Brown J. This article is distributed under the terms of the Creative Commons Attribution 4.0 International License

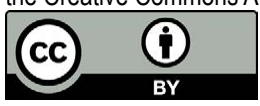

Keywords:

Traumatic Brain Injury

Mental Health

Substance Use

Intervention

Prevention
Abstract

Traumatic brain injuries (TBI) are a major community health problem in the United States. Traumatic brain injury can result in disruption to normal brain functions and is caused by a bump, blow, or external damage to the head. Ranging from mild to severe in nature, TBls can result in physical, cognitive, emotional, social, personality, adaptive, and behavioral changes in an individual. These devastating symptoms contribute to individuals with TBIs having the potential for a host of short and long-term issues. Traumatic brain injury can result in cognitive impairments, including disinhibition and risky decision-making behaviors, thus increasing the risk of substance abuse. Because many people do not have visible or physical signs, TBI can be difficult to screen, assess, and diagnose. Despite these difficulties, mental health and substance use disorder professionals can make a positive difference for clients with traumatic brain injury. As such, the current study examines the experiences and perceptions of TBI among treatment staff of Vinland National Center, a substance use disorder treatment facility in Minnesota. Results of this study raise awareness of the challenges of TBI in drug and alcohol treatment centers and offer tips, strategies, and solutions for professionals working with this clientele.

\section{Introduction}

Although the gravity of traumatic brain injuries (TBIs) often goes under-recognized and under-acknowledged, TBIs result in significant numbers of disabilities and even deaths every year in the United States and around the world (Brain Injury Association of America, 2015; Centers for Disease Control, 2015; Dewan et al., 2018; Mouzon et al., 2018). These injuries occur when a head injury causes a disruption in brain functioning. The causes of TBIs can range from falling and bumping one's head to a puncture wound of the skull. Nonetheless, not all head injuries result in TBIs. It is important to note that only head injuries that cause temporary or permanent disruptions in brain functioning are considered traumatic brain injuries. The consequences of TBIs can be classified from "mild" (i.e., short-term period of unconsciousness and/ or alterations in brain functioning) such as in the case of concussions to "severe" (i.e., lengthy period of unconsciousness and pervasive alterations in brain functioning; Jourdan, Azouvi, Genêt, Selly, Josseran, \& Schnitzler, 2018; Langlois, Rutland-Brown, \& Wald, 2006; Stambrook, Moore, Peers, Deviaene, \& Hawryluk, 1990).

Individuals with TBIs may suffer from a diverse array of physical, cognitive, and affective symptoms (for an overview of symptoms, see Appendix A). Physical symptoms include headaches, dizziness, nausea, vision problems, and sleep problems (McAllister, 2011). Cognitive symptoms include deficits in information- and languageprocessing, learning, short- and long-term memory, and decision- 
making (Chang, Hinze, Bowen, Gilbert, \& Starkey, 2018; Kinnunen et al., 2010; Poulin, Dawson, Bottari, Verreault, Turcotte, \& Jean, 2018; Schofield, Butler, Hollis, Smith, Lee, \& Kelso, 2006). Affective symptoms include emotion dysregulation (e.g., irritability, anger, and aggression; Fishbein, Dariotis, Ferguson, \& Pickelsimer, 2016; Hart, Brockway, Maiuro, Vaccaro, Fann, \& Temkin, 2017; Max, Robertson, \& Lansing, 2001), mood (e.g., depression) and anxiety issues (Malaspina et al., 2001; Reeves \& Laizer, 2012), and personality changes (Wood \& Thomas, 2013). Some research even suggests that mental health problems characterized by these affective symptoms may persist as long as 30 years, if not the rest of one's life (Koponen et al., 2002). Similarly, other research in an Australian sample of 7,485 respondents has found TBI increases risk for negative affect along with psychopathology such as anxiety and depression (Anstey, Butterworth, Jorm, Christensen, Rodgers, \& Windsor, 2004). The likelihood of all of these symptoms is exacerbated by pre-existing conditions and a history of TBIs, mental illness, and substance abuse (Schiehser, Delano-Wood, Jak, Hanson, Sorg, Orff, \& Clark, 2017; Walker, Hiller, Staton, \& Leukefeld, 2003).

One area of particular concern among individuals with TBIs is the potential for self-harm and suicide (Bahraini, Simpson, Brenner, Hoffberg, \& Schneider, 2013; Seel \& Kreutzer, 2003; Tsaousides, Cantor, \& Gordon, 2011; Wood, Williams, \& Lewis, 2010). Several studies have reported that individuals with TBIs have an elevated suicide risk relative to the general population (Brenner, Ignacio, \& Blow, 2011; Tate, Simpson, Flanagan, \& Coffey, 1997; Teasdale \& Engberg, 2001). This predisposition is related, at least in part, to the high levels of victimization observed in this population (Kim, 2002; Kwasnica \& Heinemann, 1994; Valera \& Berenbaum, 2003). For instance, youths with TBI are at risk for bullying and threats of physical harm, both of which contribute to suicidal ideation (Ilie et al., 2014). Other contributing factors to self-harm and suicidality include mental health issues (e.g., anxiety and depression; Zaninotto et al., 2016) along with sleep problems such as insomnia (Castriotta, Wilde, Lai, Atanasov, Masel, \& Kuna, 2007), which are common among individuals with TBI. As such, substance use disorder and mental health professionals should consider the potential for TBI among clients with a history of self-harm and vice-versa (Mackelprang et al., 2014).

To address this complicated web of symptomatology and co-occurring problems, routine and systematic screening and assessment for TBI is essential. This practice may hold the most promise among individuals already receiving treatment for mental health and substance use issues. Nonetheless, screening and assessment of TBI could be very challenging for at least three reasons. First, many individuals with TBI struggle with attentional control and concentration. Second, individuals with TBI are commonly unaware of their injury and its deleterious consequences.
Third, the cognitive and affective symptoms of TBI may diminish the likelihood that a client would report a TBI even when aware of its presence (Lew, Poole, Guillory, Salerno, Leskin, \& Sigford, 2006). As such, individuals with TBI are not reliable sources of information during screening and assessment. It is extremely beneficial for professionals to seek out collateral sources of information such as family members and peers along with official medical records during this process.

Once accurately diagnosed, substance use disorder and mental health professionals must tailor treatment and case management plans to the strengths and weaknesses of the client. Several rehabilitative programs including cognitive, behavioral, and educational approaches have an established body of evidence within the TBI population (Fann, Uomoto, \& Katon, 2000; Fann, Uomoto, \& Katon, 2001; Paniak, Toller-Lobe, Durand, \& Nagy, 1998). Within any of these approaches, clients with TBI tend to benefit from the use of simple and direct language, repetition, practice and role-playing, and calm and quiet environments during the course of treatment. Nonetheless, substance use disorder and mental health professionals should be prepared to deal with problematic behaviors (e.g., difficulty with comprehension and even misbehavior) during the course of treatment simply as a function of the symptoms associated with TBI. Substance use disorder and mental health professionals must stay the course with patience and understanding by reminding themselves that this is likely the result of TBI rather than malicious intentions on the part of the client.

Depending on the severity of the TBI symptoms, substance use disorder and mental health professionals may need to develop a diverse team of care providers on behalf of the client. This should likely include a professional with expertise in traumatic brain injuries and behavioral management. Expertise in neurology, neuropsychology, and psychiatry may be of interest. Other professionals that could assist in the development of skills may be beneficial. This could include independent living skills, speech therapy, and education and vocational specialists. Social work and case managers will likely be integral to recovery. The teamwork of such collectives gives individuals with TBIs the best opportunity to live independent and meaningful lives.

Because survey research in the general population has found limited awareness of TBI along with frequent misconceptions (Guilmette \& Paglia, 2004), this study sought to better understand the familiarity and everyday practices of substance use disorder and mental health professionals in regards to TBI. This survey was administered exclusively to the staff of Vinland National Center, a substance use disorder treatment facility in Minnesota. Vinland also provides educational sessions across the state of Minnesota to treatment/drug/vet courts, other treatment centers, counseling offices and 
social service and county providers on how to best identify if a potential client is working with a TBI or other cognitive deficits. Treatment professionals from Vinland were asked about their familiarity with TBI, education and training on the topic of TBI, and impressions on the treatment of clients with TBI among other questions. Findings offer insight into the current state of TBI treatment and have clear implications for the development of TBI-focused training programs.

\section{The Present Study}

This study was designed to measure the knowledge, experiences, and perceptions of professionals providing substance use disorder treatment to those impacted by traumatic brain injuries and other cognitive impairments. There is limited information available in the broader field. Further, no existing information pertaining to such professional experiences at Vinland National Center is available, as no previous research of this nature has been conducted there.

To this end, the present study has three hypotheses:

1. The level of experience will vary greatly among the staff members.

2. There is a quantifiable desire for additional TBIrelated training and materials by staff members.

3. Preferences of treatment modalities and intervention strategies will vary greatly among the staff members.

\section{Method}

Participants in the study completed an electronic survey which included single response and short answer questions. This qualitative method of inquiry allows for a predetermined set of questions to be administered to participants through a web-based survey.

\section{Procedure}

The "Perceptions of Traumatic Brain Injury (TBI) Among Professionals Providing Drug and Alcohol Addiction Treatment" survey was constructed and administered using Google Forms to capture responses by participants. Current employees of Vinland Center drug and alcohol treatment center were asked to complete the survey. No patients of the center were included in this study. A designated Vinland National Center staff member electronically distributed this survey to all staff members providing direct treatment to clients at this organization $(n=30)$. The sample size reflects the number of staff members providing direct treatment to clients at this organization. After the initial outreach, two additional survey reminders were sent out.

Prior to participating in the survey, respondents were first prompted by an informed consent page. Before proceeding with participation, all participants marked an "Agree" box prior to answering any survey questions. All survey responses were linked to a Google Sheets document for later analysis by the researchers. All aspects of this study were reviewed and approved by the Concordia University Institutional Review Board of St. Paul, Minnesota.

\section{Measure}

The survey consists of 29 questions. Featuring a variety of question formats, the survey included 15 single response questions and 14 short answer questions. Please refer to Appendix B to see a complete copy of the survey. In addition to these 29 questions, an additional 6 questions pertained to demographic variables of the respondent (i.e., gender, age, level of education, years of experience, TBI treatment certification [CBIS], and job title).

\section{Participants}

Of the 30 electronic survey requests sent, $57 \%(n=17)$ of staff members completed the survey. The respondents were $47 \%$ female $(n=8)$ and $53 \%$ male $(n=9)$. Ages were diverse with most respondents falling between the ages of 55 and $64(n=6,35 \%)$ or 35 and $44(n=5,29 \%)$. The vast majority of staff possessed an Undergraduate $(n=10,59 \%)$ or Graduate $(n=4,24 \%)$ degree. Experience levels among staff members were typically between 10 and 15 years of experience $(n=6,35 \%)$ or 5 and 10 years of experience $(n$ $=5,29 \%$ ). Of the 17 respondents, there were 15 distinct job titles listed at the Vinland facility.

Respondents were asked about their certifications and training experiences. In terms of certification, 65\% $(n=$ 11) of the respondents were Licensed Alcohol and Drug Counselors (LADC) whereas 53\% $(n=9)$ were Certified Brain Injury Specialists. Further, the vast majority of respondents $(n=13,76 \%)$ received some sort of training or education in the area of TBI. Of those who received such TBI training, respondents rated the effectiveness of this training as 3.4 on average on a scale of 1 to 5 .

\section{Results}

The survey was completed by $57 \%$ of staff members, with most having received some sort of training or education in the area of TBI. The results listed here were gathered from a number of single response and openended questions to explore the opinion and experiences of professionals providing substance use disorder treatment for individuals with brain injuries and cognitive impairments at a treatment center in Minnesota. There is no "correct" answer, and no comparison to other published studies to the researchers' knowledge related to many of the questions asked in this survey study.

Some questions asked respondents about job-related resources. For instance, in response to how often they used learning assessments on a scale of 1 to 5 , the average rating for using leaning assessments was 1.9. However, this excluded the Admissions/Intake teams, where most assessment tools are utilized in vetting potential clients' 
appropriateness levels for admittance to the treatment center. Nonetheless, the vast majority of respondents ( $n$ $=13,76 \%$ ) reported that there was access to resources to help clients with symptoms specific to TBI. Similarly, when asked how likely a participant was to refer a client with TBI to an outside mental health provider, the majority of respondents selected 76 to $100 \%(n=10,59 \%)$ or 51 to $75 \%(n=6,35 \%)$, indicating a well-established relationship with other providers in the recovery community.

A number of questions on the survey asked about the respondents' experiences with clients. One question asked, "In a typical month how many individuals do you suspect you interact with who are impacted by TBI as part of your duties as a drug and alcohol addiction treatment professional?" The most common response was 21 or more per month $(n=$ $13,76 \%)$ followed by 11 to 20 per month $(n=3,18 \%)$. Other questions asked about what percentage of clients with TBI also have histories of suicidal behaviors, co-occurring mental health disorders, and impulse control issues. For suicidal behaviors, the most common response was 0 to $25 \%(n=8$, $47 \%$ ) followed by 26 to $50 \%(n=6,35 \%)$. For co-occurring mental health disorders, the most common response was 76 to $100 \%(n=13,76 \%)$ followed by 51 to $75 \%(n=3,18 \%)$. For impulse control issues, the most common response selected was 76 to $100 \%(n=8,47 \%)$ followed by 51 to $75 \%$ $(n=4,24 \%)$. The questions below represent the responses for the open-ended questions associated with this survey:

Please describe what you see as the most important need areas for clients with traumatic brain injury (TBI) and Substance Use Disorder.
Participants were asked, "Please describe what you see as the most important need areas for clients with traumatic brain injury (TBI) and Substance Use Disorder." All responses are presented in Table 1 . As this table highlights, clients with TBIs and substance use disorders have very diverse and reaching needs that must be addressed in treatment. Foremost among the needs identified were appropriate treatment for TBI, substance use, and mental health issues. Respondents asserted that any programming must be individualized to the client's strengths and weaknesses. Beyond this, respondents viewed strong support systems as essential. These should include ongoing case management along with invested family members and friends. Other considerations identified by respondents included educational and vocational services and long-term housing.

\section{What type of relationship do you have with other providers of TBI and cognitive deficit services?}

To understand the extent of relationships of respondents with other providers, participants were asked, "What type of relationship do you have with other providers of TBI and cognitive deficit services?" Of 16 responses, 6 respondents (37.5\%) reported absent to fair relationships with such service providers. In contrast, 3 respondents described professional relationships with service providers. One respondent even described "...relationships at intake, during treatment, and at discharge to provide a continuum of care" while another respondent identified a "collaborative approach to treatment." Nonetheless, another respondent noted that this varies with each client; as some clients have

Table 1. The most important need areas for clients with traumatic brain injury and substance use disorder.

\section{Participant Responses}

Focus on mind, body and spirit and give them the tools to use these skills as part of recovery.

The success in chemical health recovery to allow client some clarity to address TBI concerns.

I believe that housing and long-term case management are the two most important needs areas.

Patience and Creativity

Programing tailored to their learning abilities

Recovery from substance use disorder (SUD) to increase mental health (MH) success

Meaningful activity - accepting communities

Information and education on how TBI and substance use impact each other.

The need for Case Management within the community.

Physical and mental health improvement

Ongoing care addressing self, family and resources

Community support

Coping skills to manage emotions and impulsivity.

Supportive long-term housing options

Their learning about the effects of the TBI on their functioning after they've been sober long enough to be sure we're seeing TBI effects. Then client needs to learn to accept and live with new abilities and limits brought about by the TBI and learn how to "work around" them in dealing with addiction and life.

Processing trauma from the TBI. Also, the loss of things they had prior to the TBI

Educated, understanding and patient providers; Education for providers and other support team members incl family members; Validation, empathy and affirmation; Safe/supportive housing; voc-rehab services; employment options; MH services. A role of mine that has emerged and become central to most Clients is to assist them explore their developed systems for getting their needs met which have built and reinforced learned patterns of behavior, both positive and negative. 
great support teams whereas others do not. Respondents mentioned established professional relationships with mental health care providers, neurologists, Assertive Community Treatment (ACT) teams, chiropractors, and legal and corrections professionals. In fact, 3 respondents (18.8\%) explicitly mentioned the Minnesota Brain Injury Alliance, which is considered a helpful source of ongoing education services and support for clients. These responses suggest a great deal of variability in the presence, quality, and scope of relationships between respondents and providers of TBI and cognitive deficit services. It also highlights the overall lack of resources specific to TBI for professionals to turn to around the state and country.

\section{What type of training do you have regarding understanding and using neuropsychological evaluation for treatment planning?}

To learn about the level of training received by participants, each was asked, "What type of training do you have regarding understanding and using neuropsychological evaluation for treatment planning?" Of 17 respondents, 9 respondents (52.9\%) reported "none" to "minimal" levels of training on the incorporation of neuropsychological tests in treatment planning. In contrast, 3 (17.6\%) respondents mentioned education via universities or seminars, 2 (11.8\%) respondents identified CBIS training, one respondent reported training in "...cognitive assessment and treatment planning," and another respondent cited working with "with neuropsychologists from Karol Neuropsychiatric Services and others throughout my 13 years." Of note, a single respondent noted using neuropsychological "...test results as a guide for medication prescribing." These findings highlight a strong need for training on how to consume and utilize neuropsychological testing in the development of treatment plans. It is important to note that Vinland employs an outside neuropsychological evaluation organization to conduct testing for clients.

\section{What learning assessments are of most benefit to you?}

Participants were also asked, "What learning assessments are of most benefit to you?" Of 17 responses, almost half of respondents $(n=7,41.2 \%)$ reported that they were unfamiliar with learning assessments or that they were not applicable. In contrast, 4 (23.5\%) respondents identified neuropsychological testing as most beneficial to them. Similarly, another 2 (11.8\%) respondents reported functional musculoskeletal assessments as beneficial to them. Other respondents identified URICA (University of Rhode Island Change Assessment Scale), MOCA (Montreal Cognitive Assessment Test Form), and MMSE (MiniMental State Examination) assessments. These responses highlight the need for greater awareness of how learning assessments can assist respondents.

\section{How do you address aggressive clients in treatment?}

A series of questions were asked to better understand the participants approach to treatment and techniques used in different situations. For instance, participants were asked, "How do you address aggressive clients in treatment?" All responses are presented in Table 2. Many responses emphasized the use of de-escalation techniques including

Table 2. Techniques to address aggressive clients during treatment.

\section{Participant Responses}

Talk with them calmly about what can be done to ease the situation.

Direct interaction and allowing client to confront their issues

Discuss coping strategies which will relieve stress

Lower tone of voice, body language that's non-threatening, safety of other clients, DO No Harm, 1\% of the time call 911

Calm approach, let them vent. Have another staff in room with me.

Managing the situation and allowing the client to feel validated

Proper de-escalation strategies / problem solving after event

De-escalation and affirmation

Talk with them, teach a mindfulness session

Motivational interviewing, active listening, find what has worked to calm clients in past, a quiet spot, mindfulness

Provide helpful outlet, such as exercise, maintain calm, and be assertive.

Listening, validating, calm voice, finding space for them

Discuss with client, teach coping skills

Help them increase awareness of what precipitates their aggressiveness, to recognize when those are emerging and intervene before an aggressive behavior emerges.

Depends on what causes the aggression and what behaviors occur when there is aggression.

Clients are provided written info regarding rules and expectations which are also explained at intake. We emphasize and model a culture of safety, respect and common courtesy. When aggressive behaviors are demonstrated, staff engage in de-escalation strategies. Clients are provided a verbal reminder of policies, norms and expectations. If behaviors occur during group Client may be asked to join case management (CM) and another staff to address with offer to dialogue/process. If there is no resolve Client may be asked to leave. We may need to assist them with disrupted transportation arrangements. We would ask Client to think about the events that informed their dismissal that day and whether they would like to return. Then they or a supportive party contact their case manager to schedule an appt to review incident and modify treatment plan. It is vital to meet with Clients for individual session asap. 
Table 3. Approaches to address low motivation during treatment.

Participant Responses

Talk with them about benefits they may receive if they give the effort.

By directly involving the client

Discuss the discrepancy between client goals and behavior, groups discussions with other stakeholders in the client's treatment.

Foster environment of "emotional safety" - so client gets somewhat emotionally connected to our program/staff. Use humor and creativity to keep things "light-hearted" throughout education in groups.

Find out/explore what intrinsically motivates the client

Directed client interaction and response

Use professional and personal supports / motivation incentives

Assigning useful, interesting tasks

Approximations towards the goal

Share potential results they could achieve if they work hard

Building purpose, hope, and active engagement

Provide a treatment plan that may address extra rest and variety of what the client may be interested in.

Motivational interviewing

Discuss with client and care providers in the community try to identify ways to motivate the client

Usually with motivational interviewing techniques such as developing discrepancy between how the client sees as the effect of his drug use and the real-life consequences the client has experienced. Asking them to explore what would be different for them if they quit using, etc.

Continue to be positive and choose small topics for the client to work on

Motivational Interviewing. Educate. Review Client goals and how investing in the process can help them achieve their goals.

teaching the client coping strategies and speaking with the client in a manner that is calm and soothing. After deescalation, some respondents identified the importance of problem-solving and increasing awareness of triggers for anger and aggression. Other techniques mentioned included the use of motivational interviewing and active listening.

\section{How do you address low motivation in treatment?}

Another topic of consideration was treatment motivation. Specifically, participants were asked, "How do you address low motivation in treatment?" Responses are presented in Table 3. A first-step offered by one respondent is to find out what intrinsically motivates the client. This can be used to discuss how treatment may benefit them. Further, multiple respondents identified the utilization of the principles and techniques of motivational interviewing. For instance, one suggested using, “...motivational interviewing techniques such as developing discrepancy between how the client sees as the effect of his drug use and the real-life consequences the client has experienced." Other approaches mentioned included the use of short and useful purpose-directed topics and tasks.

\section{How do you address comprehension issues in treatment?}

Comprehension issues during treatment are important for professionals to consider. This was gauged in this study by asking participants, "How do you address comprehension issues in treatment?" Responses are presented in Table 4. Common themes emerged in the responses including adopting a slow and methodical pace that matches the client's strengths and weaknesses. Similarly, respondents also noted the importance of using simple and straightforward language and concepts along with repetition. After discussing a topic, respondents noted the importance of ensuring that the client retained the information. This can be accomplished by asking the client to explain the concept in their own words. Another consideration is presenting any concepts in different mediums (e.g., verbal, text, picture, or role play) that match the client's strengths. Lastly, one respondent noted the importance of minimizing distractions such as noise and cell phones along with ensuring the therapeutic environment is calm.

\section{How do you address problem solving in treatment?}

Because problem solving is an essential component of treatment, all participants were asked, "How do you address problem solving in treatment?" See Table 5 for a presentation of all responses. One common approach of respondents was to discuss the problem with the client and incorporate their impressions in any resolution. Another strategy is to help clients understand the pros and cons of their decisions with real-world examples. Throughout this process, respondents recommended patience and taking a step-by-step approach. Handouts, list making, and role plays could also be beneficial.

\section{How do you assess and address cognitive deficits in treatment?}

For TBI treatment to be effective, cognitive deficits must be accounted for and addressed. To this end, all participants were asked, "How do you assess and address cognitive deficits in treatment?" See Table 6 for a presentation of all responses. There were a handful of common themes among the responses. First, two respondents identified 
Table 4. Approaches to account for low comprehension during treatment.

Participant Responses

Keep things simple and plain.

Looking for some acknowledgement from the client as to their understanding

Use modified materials.

Keep group room uncluttered/soothing (including lights), inform "what will happen" in group today, Summarize/clarify often, ask client to repeat back, repeated in a non-condescending manner when teaching, use and make memory aides, encourage them to use planner and SmartPhones

Try and keep information basic level of understanding, repeat concepts, ask clients what they recall after teaching

Working to find a sense of understanding from each client

Provide a tiered set of materials to match learning style/abilities

Assist client in understanding material

Slower pace, $1: 1$ interaction

Teach in a slow and controlled manner

Repeating the materials, more individual sessions and smaller groups

Slow and repetitive work, clients working with peers to have a variety of mannerisms/mechanisms in which information may be presented

Repetition, phrasing/presenting information in a variety of ways, checking for understanding

Offer information in a variety of forms, repetition, providing client with summaries of individual sessions, etc.

Work with the client to see what helps them better comprehend information presented, e.g., through reading, hearing, discussing, acting etc. Also assessing comprehension problems related to hearing loss, TBI, stroke, etc.

Depends on the client and what their need level is. If they need help having something explained; then it gets explained with examples. Reading assignments can be done in session

Determine capabilities v limitations through observation of their presentation while at the facility. Ever assessing. Slower pace. Repetition. Conversational style in groups. Questions encouraged.

Table 5. Approaches to problem solving during treatment.

Participant Responses

Discuss the issue and let them have some say in it.

Usually with group input

Work with client in a 1:1 setting.

Compromise and Negotiate (and state this aloud; client will invest), Get client's/group's feedback, Solution Focused Therapy, very direct/concrete, Make simple "list/notes" in 1:1's for assignments

Help clients with looking at pros/cons of decisions

Group involvement and "I" statements

I have a set of materials to help educate / practice with real world examples

Assist client in understand material

Client centered

With patience

We teach at least three different problem-solving models

Weighing options and taking things one step at a time.

Helping client to weigh out pros and cons, encourage seeking guidance and support from others, discussing cause and effect.

I am solution focused and educate clients on problems solving in both groups and individual sessions and encourage flexibility and consultation of other providers and trusted friends and family in decision making process

Ask clients about problems they've solved to get a sense of how they view their abilities to solve problems and the level of confidence they have about their ability to solve problems. Have them practice on simple problems so they can experience success and build confidence to take on bigger problems.

Make sure that each client understands what is needed for each assignment in their own way

Education/handouts/worksheets/roleplaying/multimodal approach and media. Pros and Cons work. Affirm capabilities and efforts.

utilizing repetition in treatment sessions to enhance learning. Second, three respondents considered thorough explanations and guidelines to be essential. Third, two respondents noted the importance of employing modified treatment plans for addressing cognitive deficits. Fourth, three respondents mentioned the need for neuropsychological testing and mental health referrals. Fifth, two other respondents focused on identifying and developing the client's compensatory skills and abilities. Another important contributor is the ability to have a very one-to-one relationship with each client, enhanced by each case manager having only 6 to 8 clients per caseload. This 
Table 6. Assessing and addressing cognitive deficits in treatment

\section{Participant Responses}

Education / inspiration / accountability

Provide programing at a basic level of understanding, repetitive teaching of concepts

Repetition and alternative work such as art, outdoor activities, and group work to strengthen cohesion and confidence

Thorough explanations of expectations. Make the goals achievable.

By explaining things very thoroughly

Make clear guidelines of what is expected of the clients for each assignment

Finding out what specific ways we can improve the client's ability to understand

We teach compensatory skills, and encourage to use of planners and calendars

Through questions and observation, address by modifying treatment planning

Use modified materials

Ask them what their typical days consists of (you have to get creative and dig and ask the right questions)

From the client's response

Refer for neuropsych testing and neurologist through comp assessment and meeting with client

Assess with neuropsych testing and interaction in groups and 1:1 meeting. Help client to learn to work around deficits and get out in front of them so they don't have to keep tripping over them.

Learn about if the client believes they have any deficits; recommend client get a diagnostic assessment (DA) and walk them through the process.

Refer to mental health (MH) Department

Same as above.

Table 7. Evaluating the client's success in treatment.

Participant Responses

Good attendance, participation, timely completion of assignments, either abstinence or demonstrable harm reduction substance use.

Mostly through demonstration of affecting positive personal change in their life away from treatment, in their living environment.

Completion of assignments, treatment goals, attendance and participation

Graduation and commitment to their continuing recovery treatment

Graduation from program and successful aftercare placement

By their active participation and desire for sobriety

Through increased motivation, increased engagement and participation in programming, increased insight into areas of concerns and willingness to work collaboratively with staff and trusted loved ones to address problems

"Has their overall QUALITY OF DAILY LIVING improved?", are they connected to the right resources? Do they engage socially somewhere in the community? Are they engaging socially within our program?

Stability / referrals for ongoing services / ability to engage in services

Depends on if they are trying to remain abstinent or if they are working on harm reduction; how much do they participate; are they providing insightful feedback in group, etc.

Motivations, willingness to accept continuing care

Whether the client has the tools and confidence that they can stay sober and have a life where they feel good enough about themselves to do the work necessary to stay sober.

What they are getting out of it and if they can verbalize their issues and what they learned from them

Progress towards accomplishing personal goals.

Movement towards their personal goals

According to individual progress

Post assessment upon graduation

By the post Functional Musculoskeletal Evaluation (FME)

is something Vinland does as part of its mission to serve those with TBIs and cognitive disabilities and something most mainstream treatment centers are not able to offer.

\section{How is client success evaluated in treatment?}

Another important topic is defining treatment success. To explore how success is conceptualized, all participants were asked, "How is client success evaluated in treatment?" See Table 7 for a presentation of all responses. Mentioned by six respondents, the most common approach was to incorporate some measure of treatment or program engagement. Further, three respondents focused on the progress of clients towards personal goals whereas two respondents noted graduation. The most systematic response by far noted several opportunities to measure success: "Good attendance, participation, timely completion of assignments, either abstinence or demonstrable harmreduction substance use mostly through demonstration 
Table 8. Evaluating and addressing client progress in treatment.

Participant Responses

Lack of group attendance or participation

Poor group attendance, limited participation in group programing, addressed by discussing with client what they need to be successful. If we cannot accommodate, a different treatment program may be needed.

Attendance and participation - client will get a call from their case manager and work with them to try to figure out a way to make treatment more desirable to attend and participate in.

Missing groups and little or no responses

Tracking attendance, through weekly individual sessions, discussing with outside providers, clinical team, and trusted friends and family Talk with them about pre and post results. Explain why they didn't meet goals.

Treatment plan reviews, 1:1 discussion

We will talk with them about ways to improve and possible a different approach than initially outlined.

Talk to clients about their perception of what they're doing and if they feel they are moving toward a goal of having the life they want in recovery. Ultimately to confront the reality that the client may be unable or unwilling to benefit from treatment and needs to move on.

In staffing and with their team

Discussed within clinical staff, offering multiple perspectives

Hospital admits, Housing, Detox Admits, Loss of Housing

Behavioral instability / aggression / lack of engagement after attempts to engage.

Changing treatment plan to meet client where they are, continuing to look for client's goals helping clients to reach these goals, extending length of treatment, involving clients support system

Identify barriers to progress, explore motivation for change.

If the client doesn't want to be in programming; there is an attempt to find different programming for them

See above, and, during individual sessions; weekly review of progress and Treatment Plan.

of affecting positive personal change in their life away from treatment, in their living environment." Together, these responses identify a number of viable options in operationalizing treatment success.

\section{How is the lack of client progress evaluated and addressed in treatment?}

In an effort to explore how professionals respond to lack of treatment progress, all participants were asked, "How is the lack of client progress evaluated and addressed in treatment?" See Table 8 for a presentation of all responses. The most common theme of responses focused on lack of attendance and/or participation. This was mentioned by five respondents. To address such lack of progress, four respondents stated that they would discuss performance issues with the client. For instance, one respondent replied, "We will talk with them about ways to improve and possibly a different approach than initially outlined." Beyond this, two respondents replied that they would discuss the lack of progress with their clinical staff. Other responses mentioned identifying treatment barriers, finding alternative programming, and addressing housing.

\section{What intervention(s) and strategies have you found most helpful when treating this}

population within the context of the drug and alcohol addiction treatment system?

To understand more about what treatment techniques are perceived as effective in substance use treatment settings, all participants were asked, "What intervention(s) and strategies have you found most helpful when treating this population within the context of the drug and alcohol addiction treatment system?" See Table 9 for a presentation of all responses. The most frequent response in terms of treatment approach was dialectical behavior therapy. Others mentioned included cognitive behavioral therapy and motivational interviewing. Beyond manualized treatment approaches, respondents also emphasized the use of repetition with clients and others mentioned incorporating memory aids, developing decision making, and improving overall physical health. Throughout these approaches and techniques, respondents note that individualization of treatment to the client's strengths and needs is key.

\section{What intervention(s) and strategies have you found most helpful when treating this}

population within the context of the mental health system?

To understand more about what treatment techniques are perceived as effective in mental health contexts, all participants were asked, "What intervention(s) and strategies have you found most helpful when treating this population within the context of the mental health system? Please list your responses." See Table 10 for a presentation of all responses. The most frequently mentioned treatment approach of respondents was cognitive behavioral therapy (CBT), which was identified by three respondents. Others noted the importance of case management, monitoring medication compliance, housing, and appointment reminders. Another common theme was consulting with other mental health care providers for appropriate 
Table 9. TBI treatment strategies in the context of addiction.

\section{Participant Responses}

Giving them the tools to improve their physical health

Dialectical Behavior Therapy (DBT)

\section{DBT}

Motivational Interviewing (MI), Cognitive Behavioral Therapy (CBT), Dialectical Behavior Therapy (DBT)

\section{Matrix System}

Person centered counseling, anger management tools

COLLABORATION with other team members, use consistent rules/expectations within the group dynamic

Repeating valuable information. assist client with decision making skills.

Repetition, utilizing a variety of ways of presenting the material to appeal to all learning styles, individualizing treatment plans and goals

Memory aids, repetition, motivational interviewing, being concrete, case management services, psychoeducation

Present information in very concrete, "step by step" terms to fit the client's needs and abilities to act on new information and ideas about how to live their life.

Psycho education group / coping skills training / increased supportive relationships -personal and professional.

Affirmation and redirection, understanding, empathy, reminders, being firm when needed.

The raising of awareness in dimension 4 (Readiness to Change), the teaching of relapse prevention in dimension 5 (Relapse, Continued Use, or Continued Problem Potential) and the development of a strong support system in dimension 6 (Recovery/Living Environment).

variety, strong group cohesion, and friendly non-judgmental attitude.

Exercise and Nutrition

Learned Systems Theory: Sorting out Capabilities $v$ Limitations (what they can and sometimes should do for themselves $v$ areas where they genuinely need help); regimens of validation/affirmation/redirection; group peer support, feedback and sometimes positive peer pressure. Also, education including different kinds if intelligence. Empowerment toward as much autonomy and independence as possible. Humor. Transparency. Fostering and modeling a collaborative platform.

Table 10. TBI treatment strategies in the context of mental health system.

\section{Participant Responses}

CBT

\section{CBT}

Motivational Interviewing (MI), Cognitive Behavioral Therapy (CBT)

Case management, supportive housing.

Medication compliance

Reminders of appointments and follow-ups

Talking with our fabulous mental health team.

Referring to $\mathrm{MH}$ providers, psychoeducation, teaching coping skills to manage symptoms

Regular involvement with mental health providers both therapy and psychiatry. Referrals to case management and Adult Rehabilitative Mental Health Services (ARMHS). Referring for neuropsych testing.

Kindness \& understanding when working with cognitive deficits

Patience, meeting the client where they are at

Client centered, positive approach

Educate and discuss with client "how to use their mental health therapist" more effectively. Distinguish the Myth that "therapy doesn't work".

Try to help them understand that while other conditions can often be assessed with objective measures, mental health diagnoses are mostly based on the client's mood and behavior over some period of time. Diagnosis is complicated by the use of drugs that can mask or aggravate symptoms. Further, that when clients present to mental health practitioners in a mental health crisis or while intoxicated, the client is unlikely to be a reliable reporter about his or her behavior and mood over any considerable period of time.

See above. Sometimes employing these techniques and strategies affects positive change. Licensed Alcohol and Drug Counselor (LADC) and/r Certified Brain Injury Specialist (CBIS) must remain aware that how and when we start moving into the MH arena is like wading into water; maybe going ankle deep, sometimes maybe up to calves. But then knowing when to back off and shift focus and dialogue toward exploring MH referrals.

Giving them the tools to improve their physical health NA

treatment, neuropsychological testing, and general advice. Lastly, respondents noted the importance of being kind, maintaining patience, and adopting a positive approach.

\section{Limitations}

This study had notable limitations that should be carefully considered in the context of its findings. First, 
the study had a relatively low response rate of $57 \%$ and only 17 respondents, which limits the generalizability of the findings and limits the power of the study to some extent. Additional research in broader samples including other types of facilities (e.g., forensic hospitals and detox centers), different locations (e.g., other states and countries), and more diverse samples (e.g., diversity in gender, race, and ethnicity) would be beneficial. Also, the study did not collect information on where respondents received training on $\mathrm{TBI}$, which would provide a greater knowledge of available resources in the field. Despite these limitations, this study offers an important glimpse into the services provided to individuals with TBI, which remains an understudied topic. These findings are presented as a preliminary study for future research on a larger scope.

\section{Implications}

Clients with TBIs and substance use disorders have very diverse and reaching needs that must be addressed in treatment. Foremost among the needs identified were appropriate treatment for TBI, substance use, and mental health issues. Such treatment must be individualized to the client's strengths and weaknesses and focused on relationships. There is a strong need for substance use disorder and mental health professionals to build such relationships and seek out and obtain training on how to utilize neuropsychological testing in the development of treatment plans. To address the challenges of aggression, low motivation, comprehension, problem solving, and cognitive deficits in treatment with some clients, it is important to personalize the treatment approach. Professionals must find out what intrinsically motivates the client and how treatment may benefit them. Popular treatment approaches consist of dialectical behavior therapy, cognitive behavioral therapy and motivational interviewing. Utilization of the principles and techniques of motivational interviewing can be used to point out how the client sees the effect of drug use on real-life consequences. Beyond manualized treatment approaches, the use of repetition with clients and incorporating memory aids, developing decision making, and improving overall physical health can have positive impacts on treatment success. As noted, it is critical for substance use disorder and mental health professionals to have training and experience with this population in order to identify a number of viable options in operationalizing treatment success.

\section{Recommendations for Further Research}

The current study is ripe for expansion across multiple domains. This study could be replicated in other drug and alcohol treatment facilities across the state and nation to determine if regional and cultural differences in the understanding of TBI, and its associated deficits, exist. Expansion to fields outside treatment centers may provide previously undiscovered insights into their understanding of TBI. Along the same lines, applying the survey to caregivers, immediate family members, relatives, and the client themselves may yield interesting results. Lastly, the study may provide meaningful findings if substance use disorder and mental health professionals charged with psychologically and physically evaluating clients and the impairments or deficiencies they face are surveyed. These professionals are a critical population to sample because of the multifaceted role they play in assessing and diagnosing the client and identifying treatment plans that offer the opportunity for independence and meaningful lives.

\section{Conclusion}

Traumatic brain injuries can result in substance misuse and other mental health consequences. Unfortunately, many individuals with TBI are ill-equipped to navigate the treatment system. Traumatic brain injury can cause challenges in a client's ability to understand and follow through with mainstream mental health and drug and alcohol treatment programs. Among these challenges, are problems with cognitive skills including executive functioning, emotional immaturity, and impulsiveness. These behaviors can result in actions that are counterproductive to the individual. Due to these issues and a lack of awareness on the part of those initially coming into contact with such clients (first responders, hospital staff, county programs, or even family trying to navigate the treatment systems), Vinland National Center on average does not see a client referred to them until a clients' seventh $\left(7^{\text {th }}\right)$ treatment episode. Much of this is due to the non-recognition of various neurobehavioral disorders in traditional mental health and substance use treatment programs. The need to provide highly individualized treatment is often not recognized until there have been many failed attempts at treatment.

This study contributed to the body of knowledge regarding substance use disorders, traumatic brain injury and intervention/professional development needs among treatment providers. This study explored the extent to which treatment providers at Vinland believe TBI impacts substance use disorder treatment interventions. This study also identified gaps and needs in training and professional development for working with such clients. Professionals who work with clients in a drug and alcohol treatment center are in a unique position to help individuals with traumatic brain injury. An effort to understand requirements and expectations geared to successful treatment must be imparted to each client. In order to do so, substance use disorder and mental health professionals must engage in education and training focused upon the causes, symptoms, and warning signs of TBI. Training should be focused on individualizing treatment specifically addressing TBI characteristics and an informed approach. It is imperative 
that treatment professionals recognize the importance of developing relationships and collaboration with other medical and treatment professionals to ensure adequate care for clients. With relevant awareness, education, and training among substance use disorder and mental health professionals, this population can benefit from appropriate service and support, hopefully leading to a more positive treatment outcome.

\section{Acknowledgement}

The authors would like to acknowledge the following individuals from Vinland National Center in their providing of editorial feedback on the final version of the manuscript:

Molly Gilbert, M.Ed, Director of Business Development

Tom Beckers, BIS, LADC, Residential Program Services Manager

Don Raasch, MA, LADC, CBIS, Outpatient Services Manager

\section{References}

1. Anstey, K. J., Butterworth, P., Jorm, A. F., Christensen, H., Rodgers, B., \& Windsor, T. D. (2004). A population survey found an association between self-reports of traumatic brain injury and increased psychiatric symptoms. Journal of Clinical Epidemiology, 57(11), 12021209.

2. Bahraini, N. H., Simpson, G. K., Brenner, L. A., Hoffberg, A. S., \& Schneider, A. L. (2013). Suicidal ideation and behaviours after traumatic brain injury: A systematic review. Brain Impairment, 14(1), 92-112.

3. Brain Injury Association of America, National Brain Injury Information Center. (2015). Brain injury statistics: Traumatic brain injury. Retrieved from www.biausa.org

4. Brenner, L. A., Ignacio, R. V., \& Blow, F. C. (2011). Suicide and traumatic brain injury among individuals seeking Veterans Health Administration services. The Journal of Head Trauma Rehabilitation, 26(4), 257-264.

5. Castriotta, R. J., Wilde, M. C., Lai, J. M., Atanasov, S., Masel, B. E., \& Kuna, S. T. (2007). Prevalence and consequences of sleep disorders in traumatic brain injury. Journal of Clinical Sleep Medicine, 3(04), 349356.

6. Centers for Disease Control and Prevention (2015). Traumatic brain injury in the United States: Fact sheet. Online. Available from URL www.cdc.gov/traumaticbraininjury/get_the_facts.html

7. Chang, C., Hinze, A., Bowen, J., Gilbert, L., \& Starkey, N. (2018). My memory: A mobile memory assistant for people with traumatic brain injury. International Journal of Human Computer Studies, 117, 4-19.

8. Dewan, M. C., Rattani, A., Gupta, S., Baticulon, R. E., Hung, Y. C., Punchak, M., ... \& Rosefeld, J. V. (2018). Estimating the global incidence of traumatic brain injury. Journal of Neurosurgery, 1(aop), 1-18.

9. Fann, J. R., Uomoto, J. M., \& Katon, W. J. (2001). Cognitive improvement with treatment of depression following mild traumatic brain injury. Psychosomatics, 42(1), 48-54.

10. Fann, J. R., Uomoto, J. M., \& Katon, W. J. (2000). Sertraline in the treatment of major depression following mild traumatic brain injury. The Journal of Neuropsychiatry and Clinical Neurosciences, 12(2), 226-232.

11. Fishbein, D., Dariotis, J. K., Ferguson, P. L., \& Pickelsimer, E. E. (2016) Relationships between traumatic brain injury and illicit drug use and their association with aggression in inmates. International Journal of Offender Therapy and Comparative Criminology, 60(5), 575-597.

12. Guilmette, T. J., \& Paglia, M. F. (2004). The public's misconceptions about traumatic brain injury: A follow up survey. Archives of Clinical Neuropsychology, 19(2), 183-189.

13. Hart, T., Brockway, J. A., Maiuro, R., Vaccaro, M., Fann, J., \& Temkin, N. (2017). Anger self management training for chronic moderate-severe traumatic brain injury: A randomize controlled trial. Journal of the Neurological Sciences, 381, 107-108.

14. Ilie, G., Mann, R. E., Boak, A., Adlaf, E. M., Hamilton, H., Asbridge, M., .. \& Cusimano, M. D. (2014). Suicidality, bullying and other conduct and mental health correlates of traumatic brain injury in adolescents. PloS one, 9(4), e94936.

15. Jourdan, C., Azouvi, P., Genêt, F., Selly, N., Josseran, L., \& Schnitzler A. (2018). Disability and health consequences of traumatic brain injury: National prevalence. American Journal of Physical Medicine \& Rehabilitation, 97(5), 323-331.

16. Kim, E. (2002). Agitation, aggression, and disinhibition syndromes after traumatic brain injury. NeuroRehabilitation, 17(4), 297-310.

17. Kinnunen, K. M., Greenwood, R., Powell, J. H., Leech, R., Hawkins, P. C., Bonnelle, V., ... \& Sharp, D. J. (2010). White matter damage and cognitive impairment after traumatic brain injury. Brain, 134(2), 449-463.

18. Koponen, S., Taiminen, T., Portin, R., Himanen, L., Isoniemi, H., Heinonen, H., ... \& Tenovuo, O. (2002). Axis I and II psychiatric disorders after traumatic brain injury: A 30-year follow-up study. American Journal of Psychiatry, 159(8), 1315-1321.

19. Kwasnica, C. M., \& Heinemann, A. (1994). Coping with traumatic brain injury: representative case studies. Archives of Physical Medicine and Rehabilitation, 75(4), 384-389.

20. Langlois, J. A., Rutland-Brown, W., \& Wald, M. M. (2006). The epidemiology and impact of traumatic brain injury: A brief overview. The Journal of Head Trauma Rehabilitation, 21(5), 375-378.

21. Lew, H. L., Poole, J. H., Guillory, S. B., \& Salerno, R. M. (2006). Persistent problems after traumatic brain injury: The need for long-term followup and coordinated care. Journal of Rehabilitation Research and Development, 43(2), VII.

22. Mackelprang, J. L., Bombardier, C. H., Fann, J. R., Temkin, N. R., Barber J. K., \& Dikmen, S. S. (2014). Rates and predictors of suicidal ideation during the first year after traumatic brain injury. American Journal of Public Health, 104(7), e100-e107.

23. Malaspina, D., Goetz, R. R., Friedman, J. H., Kaufmann, C. A., Faraone, S. V., Tsuang, M., ... \& Blehar, M. C. (2001). Traumatic brain injury and schizophrenia in members of schizophrenia and bipolar disorder pedigrees. American Journal of Psychiatry, 158(3), 440-446.

24. Max, J. E., Robertson, B. A. M., \& Lansing, A. E. (2001). The phenomenology of personality change due to traumatic brain injury in children and adolescents. The Journal of Neuro- psychiatry and Clinical Neurosciences, 13(2), 161-170.

25. McAllister, T. W. (2011). Neurobiological consequences of traumatic brain injury. Dialogues in Clinical Neuroscience, 13(3), 287-300.

26. Mouzon, B. C., Bachmeier, C., Ojo, J. O., Acker, C. M., Ferguson, S., Paris D., ... \& Stewart, W. (2018). Lifelong behavioral and neuropathological consequences of repetitive mild traumatic brain injury. Annals of Clinical and Translational Neurology, 5(1), 64-80.

27. Paniak, C., Toller-Lobe, G., Durand, A., \& Nagy, J. (1998). A randomized trial of two treatments for mild traumatic brain injury. Brain Injury, 12(12), 1011-1023.

28. Poulin, V., Dawson, D. R., Bottari, C., Verreault, C., Turcotte, S., \& Jean, A. (2018). Managing cognitive difficulties after traumatic brain injury: A review of online resources for families. Disability and Rehabilitation, $1-11$. 
29. Reeves, R. R., \& Laizer, J. T. (2012). Traumatic brain injury and suicide. Journal of Psychosocial Nursing and Mental Health Services, 50(3), 32-38.

30. Schiehser, D. M., Delano-Wood, L., Jak, A. J., Hanson, K. L., Sorg, S. F., Orff, H., \& Clark, A. L. (2017). Predictors of cognitive and physical fatigue in post-acute mild-moderate traumatic brain injury. Neuropsychological Rehabilitation, 27(7), 1031-1046.

31. Schofield, P. W., Butler, T. G., Hollis, S. J., Smith, N. E., Lee, S. J., \& Kelso, W. M. (2006). Neuropsychiatric correlates of traumatic brain injury (TBI) among Australian prison entrants. Brain Injury, 20(13-14), 1409-1418.

32. Seel, R. T., \& Kreutzer, J. S. (2003). Depression assessment after traumatic brain injury: An empirically based classification method Archives of Physical Medicine and Rehabilitation, 84(11), 1621-1628.

33. Stambrook, M., Moore, A. D., Peters, L. C., Deviaene, C., \& Hawryluk, G. A. (1990). Effects of mild, moderate and severe closed head injury on long-term vocational status. Brain Injury, 4(2), 183-190.

34. Tate, R., Simpson, G., Flanagan, S., \& Coffey, M. (1997). Completed suicide after traumatic brain injury. The Journal of Head Trauma Rehabilitation, 12(6), 16-28.

35. Teasdale, T. W., \& Engberg, A. W. (2001). Suicide after traumatic brain injury: A population study. Journal of Neurology, Neurosurgery \& Psychiatry, 71(4), 436-440.

36. Tsaousides, T., Cantor, J. B., \& Gordon, W. A. (2011). Suicidal ideation following traumatic brain injury: Prevalence rates and correlates in adults living in the community. The Journal of Head Trauma Rehabilitation, 26(4), 265-275.

37. Valera, E. M., \& Berenbaum, H. (2003). Brain injury in battered women Journal of Consulting and Clinical Psychology, 71(4), 797.

38. Walker, R., Hiller, M., Staton, M., \& Leukefeld, C. G. (2003). Head injury among drug abusers: An indicator of co-occurring problems. Journal of Psychoactive Drugs, 35(3), 343-353.

39. Wood, R. L., \& Thomas, R. H. (2013). Impulsive and episodic disorders of aggressive behavior following traumatic brain injury. Brain Injury, 27(3), 253-261.

40. Wood, R. L., Williams, C., \& Lewis, R. (2010). Role of alexithymia in suicide ideation after traumatic brain injury. Journal of the International Neuropsychological Society, 16(6), 1108-1114.

41. Zaninotto, A. L., Vicentini, J. E., Fregni, F., Rodrigues, P. A., Botelho, C., de Lucia, M. C. S., \& Paiva, W. S. (2016). Updates and current perspectives of psychiatric assessments after traumatic brain injury: A systematic review. Frontiers in Psychiatry, 7, 95 


\section{Appendix A}

Specific Consequences Associated with TBI Based on a Review of Peer-reviewed Literature.

\begin{tabular}{|l|l|}
\hline Symptom Category & Specific Examples of TBI Consequences \\
\hline Affective & Flat Affect, Mood Swings, and Uncontrolled Laughing and/or Crying \\
\hline Behavioral & Aggression and Impulsivity \\
\hline Cognitive & Confusion, Information-Processing Deficits, Memory Impairments, and Organization and Planning Deficits \\
\hline Communication & Language and Speech Deficits, Misperception of Social Cues, and Social Skills \\
\hline Physical & $\begin{array}{l}\text { Agitation, Chronic Body Pain, Dizziness, Fatigue, Headaches, Mobility Limitations, Nausea, and Sleep Disor- } \\
\text { ders }\end{array}$ \\
\hline Sensory & Visual-Spatial Impairments, Sensory Integration Problems, and Loss of Sense of Smell \\
\hline
\end{tabular}

\section{Appendix B.}

Survey Questions

1. Please describe what you see as the most important need areas for clients with traumatic brain injury (TBI) and Substance Use Disorder.

2. What type of relationship do you have with other providers of TBI and cognitive deficit services?

3. Have you ever received training (education) related to TBI? Choose one response.

4. If you have received training on the recognition of TBI, how useful was it in helping you work with someone with Substance Use Disorder and TBI? Choose one response.

5. Are you certified brain injury specialist (CBIS) certified?

6. How many years have you been working in the field of drug and alcohol addiction treatment? Choose one response.

7. In a typical month how many individuals do you suspect you interact with who are impacted by TBI as part of your duties as a drug and alcohol addiction treatment professional? Choose one response.

8. In your experience what \% of client's with TBI also have a history of suicidal behaviors? Choose one response.

9. In your experience what \% of client's with TBI also have one or more co-occurring mental health disorders? Choose one response.

10. What \% of your client's with TBI demonstrates impulse control issues? Choose one response.

11. What type of training do you have regarding understanding and using neuropsychological evaluation for treatment planning?

12. How often do you use learning assessments?

13. What learning assessments are of most benefit to you?

14. How often do you address memory impairments in treatment?

15. Do you possess the resources to help clients with symptoms specific to TBI such as impulsivity, anger, memory deficits etc.?

16. How do you address aggressive clients in treatment?

17. How do you address low motivation in treatment?

18. How do you address comprehension issues in treatment?

19. How do you address problem solving in treatment?

20. How do you assess and address cognitive deficits in treatment?

21. How is client success evaluated in treatment?

22. How is lack of client progress evaluated and addressed in treatment?

23. How likely are you to refer a patient with TBI to an outside mental health provider? Choose one response.

24. What intervention(s) and strategies have you found most helpful when treating this population within the context of the drug and alcohol addiction treatment system?

25. What intervention(s) and strategies have you found most helpful when treating this population within the context of the mental health system? Please list your responses.

26. What is your gender? Choose one response.

27. Please indicate where your age falls in the ranges below? Choose one response.

28. What is your highest completed degree level? Choose one response.

29. What is your (general) role at Vinland? Please briefly describe licensure. 